\title{
Universal feedback control of two-qubit entanglement
}

\author{
Morteza Rafiee, ${ }^{1, *}$ Alireza Nourmandipour, ${ }^{2, \dagger}$ and Stefano Mancini ${ }^{3,4, \ddagger}$ \\ ${ }^{1}$ Department of Physics, Shahrood University of Technology, 3619995161 Shahrood, Iran \\ ${ }^{2}$ Atomic and Molecular Group, Faculty of Physics, Yazd University, Yazd 89195-741, Iran \\ ${ }^{3}$ School of Science and Technology, University of Camerino, I-62032 Camerino, Italy \\ ${ }^{4}$ INFN-Sezione di Perugia, Via A. Pascoli, I-06123 Perugia, Italy
}

(Received 7 April 2017; published 31 July 2017)

\begin{abstract}
We consider two qubits undergoing local dissipation and subject to local driving. We then determine the optimal Markovian feedback action to preserve initial entanglement as well as to create stationary entanglement with the help of an XY interaction Hamiltonian. Such feedback actions are worked out in a way not depending on the initial two-qubit state, whence called universal.
\end{abstract}

DOI: 10.1103/PhysRevA.96.012340

\section{INTRODUCTION}

Quantum entanglement has been recognized in recent decades as a resource for quantum information processing [1]. As such, it should be controllable. Several efforts have been devoted to control entanglement [2]. Control can take place with open-loop or and closed-loop strategies according to the principle of controllers design [3]. Quite generally, closed-loop control performs better than open-loop control because it involves gathering information about the system state and then, according to that, actuates a corrective action on its dynamics, but results are more difficult to implement [4]. A good compromise between these two tensions is probably represented by Markovian feedback [5], which brings the advantages of closed-loop control but is not very difficult to realize. In fact, it rests on an actuation based on the measurement result obtained immediately before, hence the name Markovian. Nevertheless, it carries an inherent double optimization, over the measurement and over the actuation [6]. This makes designing optimal control a daunting task even for Markovian feedback, especially when dealing with composite systems and hence with entanglement control (we refer here to local control, i.e., measurement and actuation are both local operations). The best results (in terms of optimality) have been achieved in the context of Gaussian systems [7]. For qubit systems, due to their inherent nonlinearity, the situation is more complicated. With two qubit, on the one hand, a proof of principle of the effectiveness of Markovian feedback in stabilizing entanglement was given in [8], but it is not optimal. On the other hand, the effectiveness of Markovian feedback in protecting initial entangled states has been shown in [9]. This action, though optimal, was derived in a way depending on the initial state.

Here we generalize these results by determining the optimal Markovian feedback action to preserve initial entanglement as well as to create stationary entanglement with the help of an XY interaction Hamiltonian. Moreover, such feedback actions are worked out in a way not depending on the initial two-qubit state, whence referred to as universal.

\footnotetext{
*m.rafiee178@gmail.com

†anoormandip@stu.yazd.ac.ir

${ }^{\ddagger}$ stefano.mancini@unicam.it
}

The layout of the paper is as follows. We start by introducing the model feedback action in Sec. II. Then we address the issue of preserving initial entanglement in Sec. III and subsequently the issue of stabilizing entanglement in Sec. IV. Finally, Sec. V is the conclusion. Throughout the paper we will use $\iota$ to denote the imaginary unit.

\section{THE MODEL}

Consider a two-qubit system whose dynamics is governed by the following master equation:

$$
\dot{\rho}=-\iota[H, \rho]+\mathcal{D}\left[\sigma_{1}\right] \rho+\mathcal{D}\left[\sigma_{2}\right] \rho,
$$

where $H$ denotes the Hamiltonian and $\sigma_{i}$ and $\sigma_{i}^{\dagger}(i=1,2)$ are the lowering and raising Pauli operators, respectively. Furthermore,

$$
\mathcal{D}[c] \rho \equiv c \rho c^{\dagger}-\frac{1}{2} c^{\dagger} c \rho-\frac{1}{2} \rho c^{\dagger} c
$$

is the dissipative superoperator and the way it appears in Eq. (1) shows qubit dissipation into local environments.

The master equation (1) can be unraveled by stochastic equations driven either by point processes or by Wiener processes (Gaussian white noise) [6]. Different unraveling corresponds to different kinds of measurements. A point process consists of intervals of deterministic (smooth) motion, interleaved by instantaneous events in which the state of the system changes. In particular, following the reasoning of Ref. [5] and generalizing it, we may consider selective evolutions under local point processes with probability operator value elements

$$
\begin{aligned}
& \Omega_{1}(d t)=\sqrt{d t} \sigma_{1} \\
& \Omega_{2}(d t)=\sqrt{d t} \sigma_{2}, \\
& \Omega_{3}(d t)=1-\left(\iota H+\frac{1}{2} \sigma_{1}^{\dagger} \sigma_{1}+\frac{1}{2} \sigma_{2}^{\dagger} \sigma_{2}\right) d t
\end{aligned}
$$

describing detection (jump) on the first (respectively, second) environment $\Omega_{1}$ (respectively, $\Omega_{2}$ ) and no detection $\Omega_{3}$. The measurement time is the infinitesimal $d t$ as it is appropriate for continuous measurement. This corresponds to local excitation detection of qubits, namely, measuring $\sigma_{i}^{\dagger} \sigma_{i}-I$. 
It is then easy to verify that the nonselective evolution under this measurement

$$
\rho(t+d t)=\sum_{j=1}^{3} \Omega_{j}(d t) \rho(t) \Omega_{j}^{\dagger}(d t),
$$

is equivalent to the master equation (1).

The selective evolution allows us to incorporate the feedback action. This one, in order to be Markovian, must cause an immediate state change based only on the result of the measurement in the preceding infinitesimal time interval. Hence it must occur immediately after a detection and cause a finite amount of evolution.

Let this finite evolution following a detection on qubit $i$ at time $t$ be determined by a Liouville superoperator $\mathcal{K}_{i}$, then

$$
\tilde{\rho}_{i}(t+d t)=e^{\mathcal{K}_{i}}\left[\Omega_{i}(d t) \rho(t) \Omega_{i}^{\dagger}(d t)\right]
$$

where the tilde means that the density operator is unnormalized. From Eq. (5) it is clear that the feedback action is local.

The nonselective evolution of the system is then given by

$$
\rho(t+d t) \propto \tilde{\rho}_{1}(t+d t)+\tilde{\rho}_{2}(t+d t)+\tilde{\rho}_{3}(t+d t),
$$

where $\tilde{\rho}_{3}(t+d t)=\Omega_{3}(d t) \rho(t) \Omega_{3}^{\dagger}(d t)$. Since the latter is unchanged by feedback, we get for the normalized density operator

$$
\dot{\rho}=-\iota[H, \rho]+\sum_{i=1}^{2}\left[e^{\mathcal{K}_{i}} \sigma_{i} \rho \sigma_{i}^{\dagger}-\frac{1}{2} \sigma_{i}^{\dagger} \sigma_{i} \rho-\frac{1}{2} \rho \sigma_{i}^{\dagger} \sigma_{i}\right] .
$$

Assuming that $\mathcal{K}_{i}$ 's act in a Hamiltonian way (so to avoid introducing further noise),

$$
\mathcal{K}_{i} \rho=-\iota\left[F_{i}, \rho\right]
$$

we will further get

$$
\dot{\rho}=-\iota[H, \rho]+\mathcal{D}\left[e^{-\iota F_{1}} \sigma_{1}\right] \rho+\mathcal{D}\left[e^{-\iota F_{2}} \sigma_{2}\right] \rho,
$$

where $F_{1}, F_{2}$ are Hermitian operators on $\mathbb{C}^{2}$.

In contrast, unraveling master equation (1) by stochastic equations driven by Wiener processes (Gaussian white noise) corresponds to measurement techniques that are quite different from excitation detection, e.g., measuring $\sigma_{i}^{\dagger} \zeta-\zeta^{*} \sigma_{i}$, with $\zeta \in \mathbb{C}$. Nevertheless, the feedback master equation can be derived from (9) by letting

$$
\begin{aligned}
& H \rightarrow H+\iota \frac{1}{2}\left(\sigma_{1}^{\dagger} \zeta-\zeta^{*} \sigma_{1}+\sigma_{2}^{\dagger} \zeta-\zeta^{*} \sigma_{2}\right)-\zeta\left(F_{1}+F_{2}\right), \\
& \mathcal{D}\left[e^{-\iota F_{i}} \sigma_{i}\right] \rightarrow \mathcal{D}\left[e^{-\iota F_{i} / \zeta}\left(\sigma_{i}+\zeta\right)\right],
\end{aligned}
$$

then expanding to second order in $1 /|\zeta|$ and finally taking the limit $|\zeta| \rightarrow \infty$. As such, it can be considered as a special case of the feedback with point process. Therefore, for the sake of generality, hereafter we focus our attention on the latter.

Operators $F_{i}$ in (9) play the role of (local) feedback Hamiltonians and they concur to implement unitary local actuations $e^{-l F_{i}}$. Hence these latter can be parametrized as follows:

$$
e^{-l F_{i}}=\left(\begin{array}{cc}
e^{-l\left(\alpha_{i}+\gamma_{i}\right) / 2} \cos \left(\beta_{i} / 2\right) & -e^{-\iota\left(\alpha_{i}-\gamma_{i}\right) / 2} \sin \left(\beta_{i} / 2\right) \\
e^{l\left(\alpha_{i}-\gamma_{i}\right) / 2} \sin \left(\beta_{i} / 2\right) & e^{l\left(\alpha_{i}+\gamma_{i}\right) / 2} \cos \left(\beta_{i} / 2\right)
\end{array}\right),
$$

with $0 \leqslant \alpha_{i}, \beta_{i}, \gamma_{i} \leqslant 2 \pi(i=1,2)$.
Furthermore, it is

$$
\begin{aligned}
e^{-\iota F_{i}} \sigma_{i}= & \left(\begin{array}{ll}
-e^{-\iota\left(\alpha_{i}-\gamma_{i}\right) / 2} \sin \left(\beta_{i} / 2\right) & 0 \\
e^{\iota\left(\alpha_{i}+\gamma_{i}\right) / 2} \cos \left(\beta_{i} / 2\right) & 0
\end{array}\right) \\
= & -e^{-\iota\left(\alpha_{i}-\gamma_{i}\right) / 2} \sin \left(\beta_{i} / 2\right)|1\rangle_{i}\langle 1| \\
& +e^{\iota\left(\alpha_{i}+\gamma_{i}\right) / 2} \cos \left(\beta_{i} / 2\right)|0\rangle_{i}\langle 1|,
\end{aligned}
$$

having assumed $|1\rangle_{i}$ as the excited state and $|0\rangle_{i}$ as the ground state of the $i$ th qubit.

Quite generally we can split the Hamiltonian $H$ into two contributions: a local driving term, e.g.,

$$
H_{\text {drive }}=\iota \alpha\left(\sigma_{1}-\sigma_{1}^{\dagger}\right)+\iota \alpha\left(\sigma_{2}-\sigma_{2}^{\dagger}\right)
$$

with driving amplitude $\alpha \in \mathbb{R}$ and an interaction term $H_{\text {int }}$ to be specified.

Then Eq. (9) explicitly becomes

$$
\begin{aligned}
\dot{\rho}= & -\iota\left[\alpha \sigma_{1}^{(y)}+\alpha \sigma_{2}^{(y)}, \rho\right]-\iota\left[H_{\mathrm{int}}, \rho\right] \\
& -\sum_{i=1}^{2}\left(\frac{1}{2}\left(\left|A_{i}\right|^{2}+\left|B_{i}\right|^{2}\right)\left(|1\rangle_{i}\langle 1|\rho+\rho| 1\rangle_{i}\langle 1|\right)\right. \\
& \left.+\left(A_{i}|1\rangle_{i}\left\langle 1\left|+B_{i}\right| 0\right\rangle_{i}\langle 1|\right) \rho\left(A_{i}^{*}|1\rangle_{i}\left\langle 1\left|+B_{i}\right| 1\right\rangle_{i}\langle 0|\right)\right),
\end{aligned}
$$

with

$$
\begin{aligned}
A_{i} & =-e^{-\iota\left(\alpha_{i}-\gamma_{i}\right) / 2} \sin \left(\beta_{i} / 2\right), \\
B_{i} & =e^{\iota\left(\alpha_{i}+\gamma_{i}\right) / 2} \cos \left(\beta_{i} / 2\right) .
\end{aligned}
$$

This means that $\left|A_{i}\right|^{2}+\left|B_{i}\right|^{2}=1$.

At this point the aim would be the optimization of a measure of entanglement over the parameters characterizing $F_{1}$ and $F_{2}$, or equivalently $e^{-\iota F_{1}}$ and $e^{-\iota F_{2}}$.

The figure of merit we shall employ for entanglement is the concurrence defined as [10]

$$
C(\rho):=\max \left\{0, \lambda_{1}-\lambda_{2}-\lambda_{3}-\lambda_{4}\right\},
$$

where $\lambda_{i}$ 's are, in decreasing order, the non-negative square roots of the moduli of the eigenvalues of

$$
\rho\left(\sigma_{1}-\sigma_{1}^{\dagger}\right) \otimes\left(\sigma_{2}-\sigma_{2}^{\dagger}\right) \rho^{*}\left(\sigma_{1}-\sigma_{1}^{\dagger}\right) \otimes\left(\sigma_{2}-\sigma_{2}^{\dagger}\right) .
$$

Furthermore, in the following we will distinguish two tasks: entanglement preservation and entanglement stabilization.

\section{PRESERVING ENTANGLEMENT}

Suppose we want to preserve as much as possible an initial entangled state by using feedback and considering $H_{\text {int }}=0$ in Eq. (14). Then, in the basis $\{|11\rangle,|10\rangle,|01\rangle,|00\rangle\}$ such equation 
becomes

$$
\begin{aligned}
\dot{\rho}= & -\iota\left(\begin{array}{cccc}
0 & -\iota \alpha & -\iota \alpha & 0 \\
\iota & 0 & 0 & -\iota \alpha \\
\iota \alpha & 0 & 0 & -\iota \alpha \\
0 & \iota \alpha & \iota \alpha & 0
\end{array}\right) \rho+\iota \rho\left(\begin{array}{cccc}
0 & -\iota \alpha & -\iota \alpha & 0 \\
\iota \alpha & 0 & 0 & -\iota \alpha \\
\iota \alpha & 0 & 0 & -\iota \alpha \\
0 & \iota \alpha & \iota \alpha & 0
\end{array}\right) \\
& +\left(\begin{array}{cccc}
A_{1} & 0 & 0 & 0 \\
0 & A_{1} & 0 & 0 \\
B_{1} & 0 & 0 & 0 \\
0 & B_{1} & 0 & 0
\end{array}\right) \rho\left(\begin{array}{cccc}
A_{1}^{*} & 0 & B_{1}^{*} & 0 \\
0 & A_{1}^{*} & 0 & B_{1}^{*} \\
0 & 0 & & 0 \\
0 & 0 & 0 & 0
\end{array}\right) \\
& +\left(\begin{array}{cccc}
A_{2} & 0 & 0 & 0 \\
B_{2} & 0 & 0 & 0 \\
0 & 0 & A_{2} & 0 \\
0 & 0 & B_{2} & 0
\end{array}\right) \rho\left(\begin{array}{cccc}
A_{2}^{*} & B_{2}^{*} & & 0 \\
0 & 0 & 0 & 0 \\
0 & 0 & A_{2}^{*} & B_{2}^{*} \\
0 & 0 & 0 & 0
\end{array}\right) \\
& -\frac{1}{2}\left(\begin{array}{cccc}
2 & 0 & 0 & 0 \\
0 & 1 & 0 & 0 \\
0 & 0 & 1 & 0 \\
0 & 0 & 0 & 0
\end{array}\right) \rho-\frac{1}{2} \rho\left(\begin{array}{cccc}
2 & 0 & 0 & 0 \\
0 & 1 & 0 & 0 \\
0 & 0 & 1 & 0 \\
0 & 0 & 0 & 0
\end{array}\right) .
\end{aligned}
$$

Writing

$$
\rho=\left(\begin{array}{cccc}
\mathcal{A} & \mathcal{B}_{R}+\iota \mathcal{B}_{I} & \mathcal{C}_{R}+\iota \mathcal{C}_{I} & \mathcal{D}_{R}+\iota \mathcal{D}_{I} \\
\mathcal{B}_{R}-\iota \mathcal{B}_{I} & \mathcal{E} & \mathcal{F}_{R}+\iota \mathcal{F}_{I} & \mathcal{G}_{R}+\iota \mathcal{G}_{I} \\
\mathcal{C}_{R}-\iota \mathcal{C}_{I} & \mathcal{F}_{R}-\iota \mathcal{F}_{I} & \mathcal{H} & \mathcal{I}_{R}+\iota \mathcal{I}_{I} \\
\mathcal{D}_{R}-\iota \mathcal{D}_{I} & \mathcal{G}_{R}-\iota \mathcal{G}_{I} & \mathcal{I}_{R}-\iota \mathcal{I}_{I} & 1-\mathcal{A}-\mathcal{E}-\mathcal{H}
\end{array}\right),
$$

Eq. (18) can be put in the following form:

$$
\dot{\mathbf{v}}=\mathbf{M v}-\mathbf{w},
$$

where $\mathbf{v}$ is the unknown vector

$$
\mathbf{v}:=\left(\mathcal{A}, \mathcal{B}_{R}, \mathcal{B}_{I}, \mathcal{C}_{R}, \mathcal{C}_{I}, \mathcal{D}_{R}, \mathcal{D}_{I}, \mathcal{E}, \mathcal{F}_{R}, \mathcal{F}_{I}, \mathcal{G}_{R}, \mathcal{G}_{I}, \mathcal{H}, \mathcal{I}_{R}, \mathcal{I}_{I}\right)
$$

with entries depending on time $t$, while

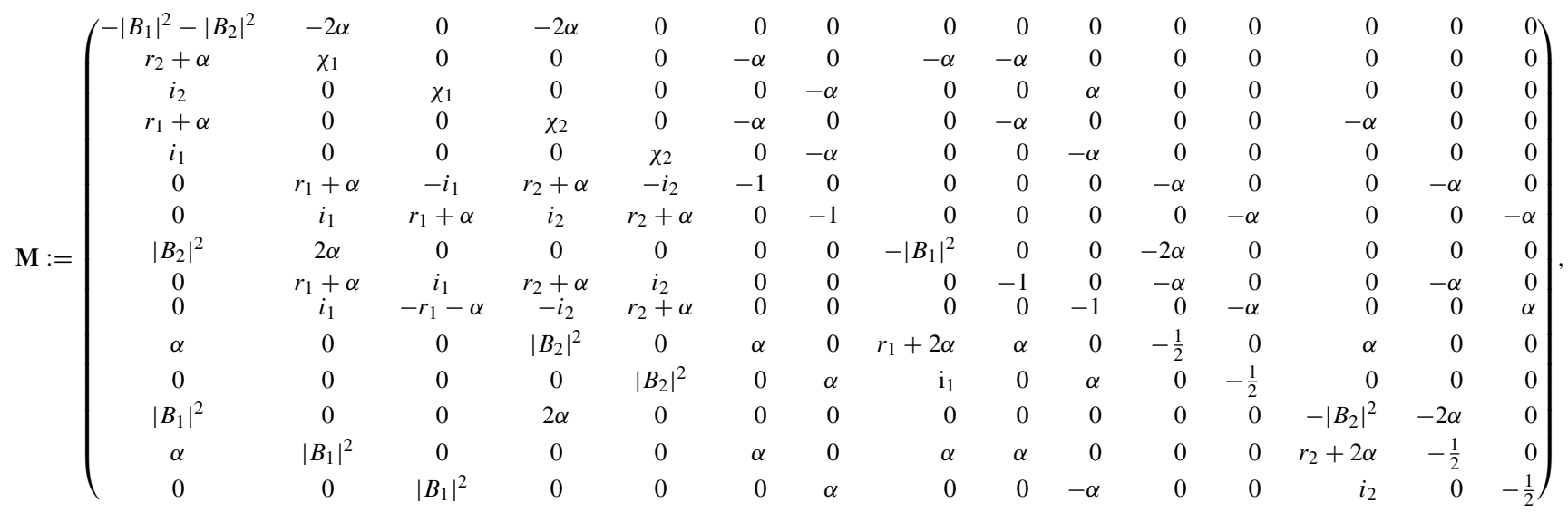

with

$$
\begin{aligned}
r_{i} & =\operatorname{Re}\left[A_{i} B_{i}^{*}\right], \\
i_{i} & =\operatorname{Im}\left[A_{i} B_{i}^{*}\right], \\
\chi_{i} & =-\left(\frac{3}{2}-\left|A_{i}\right|^{2}\right),
\end{aligned}
$$

and

$$
\mathbf{w}:=\left(\begin{array}{lllllllllllllll}
0 & 0 & 0 & 0 & 0 & 0 & 0 & 0 & 0 & 0 & \alpha & 0 & 0 & \alpha & 0
\end{array}\right)^{\top} .
$$

Notice that Eq. (20), thanks to (22), results independent of $\gamma_{i}$ 's. 


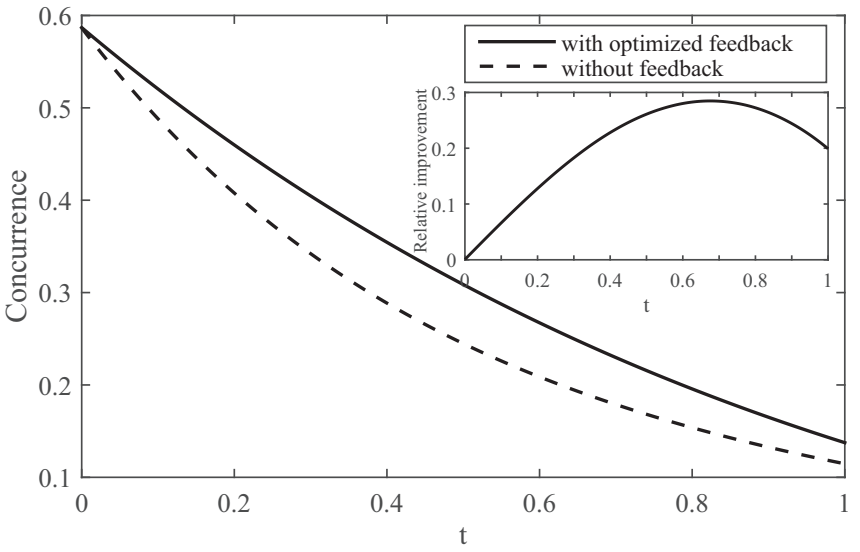

FIG. 1. Entanglement measured by concurrence versus (dimensionless) time with and without feedback. Inset: Relative improvement of concurrence due to feedback versus (dimensionless) time.

Let the initial condition be

$$
\rho(0)=|\Psi\rangle\langle\Psi|,
$$

with a generic pure state $|\Psi\rangle$ parametrized as

$$
\begin{aligned}
|\Psi\rangle= & \cos \theta_{3}|11\rangle+e^{\iota \phi_{2}} \cos \theta_{1} \sin \theta_{3} \sin \theta_{2}|01\rangle \\
& +e^{\iota \phi_{1}} \cos \theta_{2} \sin \theta_{3}|10\rangle+e^{\iota \phi_{3}} \sin \theta_{3} \sin \theta_{2} \sin \theta_{1}|00\rangle,
\end{aligned}
$$

being $\theta_{i} \in[0, \pi / 2]$ and $\phi_{i} \in[0,2 \pi)$. In turn, this means

$$
\begin{aligned}
\mathcal{A}(0) & =\cos ^{2} \theta_{3}, \\
\mathcal{B}_{R}(0) & =\cos \phi_{1} \cos \theta_{3} \cos \theta_{2} \sin \theta_{3}, \\
\mathcal{B}_{I}(0) & =-\sin \phi_{1} \cos \theta_{3} \cos \theta_{2} \sin \theta_{3}, \\
\mathcal{C}_{R}(0) & =\cos \phi_{2} \cos \theta_{3} \cos \theta_{1} \sin \theta_{3} \sin \theta_{2}, \\
\mathcal{C}_{I}(0) & =-\sin \phi_{2} \cos \theta_{3} \cos \theta_{1} \sin \theta_{3} \sin \theta_{2}, \\
\mathcal{D}_{R}(0) & =\cos \phi_{3} \cos \theta_{3} \sin \theta_{3} \sin \theta_{2} \sin \theta_{1}, \\
\mathcal{D}_{I}(0) & =-\sin \phi_{3} \cos \theta_{3} \sin \theta_{3} \sin \theta_{2} \sin \theta_{1}, \\
\mathcal{E}(0) & =\cos \theta_{2} \sin ^{2} \theta_{3}, \\
\mathcal{F}_{R}(0) & =\cos \left(\phi_{1}-\phi_{2}\right) \cos \theta_{2} \cos \theta_{1} \sin ^{2} \theta_{3} \sin \theta_{2}, \\
\mathcal{F}_{I}(0) & =\sin \left(\phi_{1}-\phi_{2}\right) \cos \theta_{2} \cos \theta_{1} \sin ^{2} \theta_{3} \sin \theta_{2}, \\
\mathcal{G}_{R}(0) & =\cos \left(\phi_{1}-\phi_{3}\right) \cos \theta_{2} \sin ^{2} \theta_{3} \sin \theta_{2} \sin \theta_{1}, \\
\mathcal{G}_{I}(0) & =\sin \left(\phi_{1}-\phi_{3}\right) \cos \theta_{2} \sin ^{2} \theta_{3} \sin _{2} \sin \theta_{1}, \\
\mathcal{H}(0) & =\cos \theta^{2} \theta_{1} \sin ^{2} \theta_{3} \sin \theta^{2} \theta_{2}, \\
\mathcal{I}_{R}(0) & =\cos \left(\phi_{2}-\phi_{3}\right) \cos \theta_{1} \sin ^{2} \theta_{3} \sin ^{2} \theta_{2} \sin \theta_{1}, \\
\mathcal{I}_{I}(0) & =\sin \left(\phi_{2}-\phi_{3}\right) \cos \theta_{1} \sin ^{2} \theta_{3} \sin ^{2} \theta_{2} \sin \theta_{1} .
\end{aligned}
$$

TABLE I. Values of parameters $\alpha_{i}, \beta_{i}$ realizing optimal feedback action.

\begin{tabular}{lccc}
\hline \hline$\alpha_{1}$ & $\beta_{1}$ & $\alpha_{2}$ & $\beta_{2}$ \\
\hline$\pi / 6$ & $5 \pi / 6$ & $5 \pi / 6$ & $\pi$ \\
\hline \hline
\end{tabular}

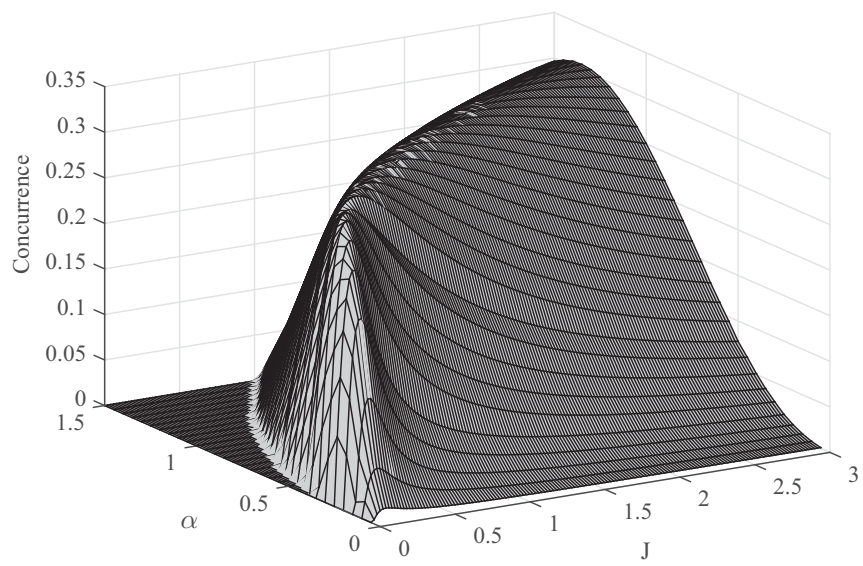

FIG. 2. Concurrence achieved with optimal feedback vs $\alpha$ and $J$.

Clearly depending on the values of parameters $\theta_{i}$ and $\phi_{i}$ the initial state can be entangled or factorable. However, a randomly chosen set of parameters almost surely gives an entangled state [11]. Hence, we will consider the concurrence at a given time $C(\rho(t))$ averaged over all possible initial states and then maximize it over $\alpha_{i}, \beta_{i}$. This will lead to a universal control action, i.e., independent of the initial state. To this end initial states are chosen according to the following measure induced by Haar measure on U(4) [12]:

$$
d \mu(|\Psi\rangle)=\frac{6}{\pi^{3}} \prod_{i=1}^{3} \cos \theta_{i}\left(\sin \theta_{i}\right)^{(2 i-1)} d \theta_{i} d \phi_{i} .
$$

Actually, it is useful to consider

$$
\theta_{i}:=\arcsin \left(\xi_{i}^{1 /(2 i)}\right),
$$

so to have flat probability densities for $\phi_{i} \in[0,2 \pi]$ and $\xi_{i} \in$ $[0,1]$,

$$
P\left(\phi_{i}\right)=\frac{1}{2 \pi}, \quad P\left(\xi_{i}\right)=1 .
$$

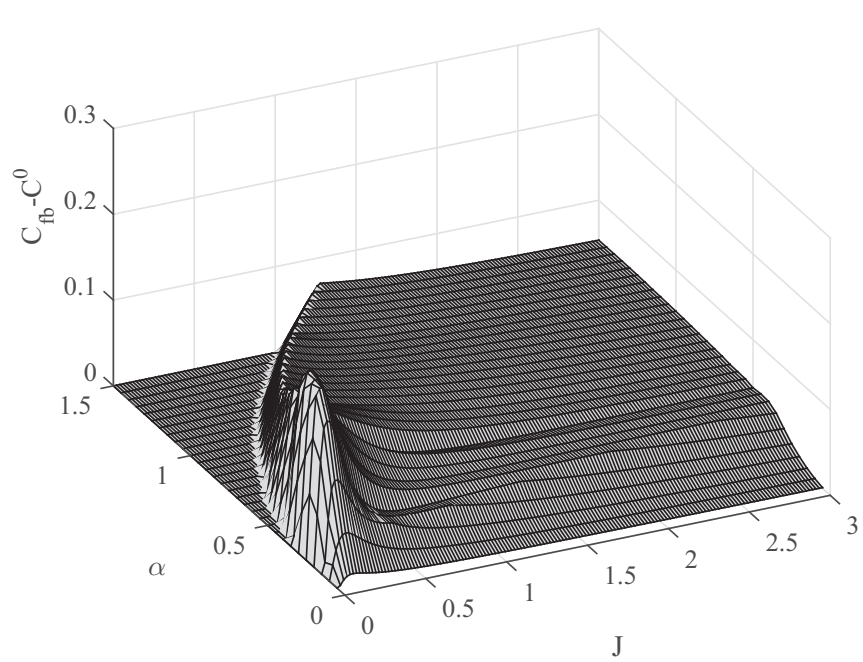

FIG. 3. Difference between concurrence achieved with optimal feedback and concurrence achieved without feedback vs $\alpha$ and $J$. 


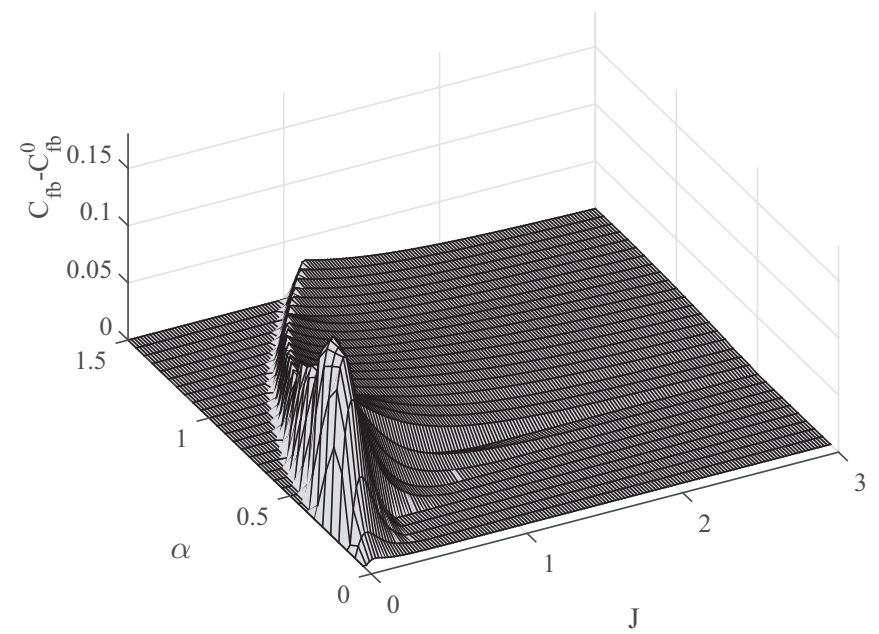

FIG. 4. Difference between concurrence achieved with optimal feedback and concurrence achieved with feedback as in Ref. [8] vs $\alpha$ and $J$.

Equation (20) is solved analytically (not reported here for the sake of simplicity) subjected to the initial condition (27). Then the average concurrence has been calculated numerically at each time $t$ using an ensemble of $10^{5}$ states. The procedure is repeated for values of $\alpha_{i}, \beta_{i}$ in the range $0,2 \pi$ with step $\pi / 12$. Finally, the maximum value is taken as corresponding to optimal feedback and the value $\alpha_{i}=\beta_{i}=0$ is taken as corresponding to no feedback action.

The remarkable thing is that the average concurrence does not depend on the driving parameter $\alpha$. Then the results comparing the average concurrence with optimal feedback and without feedback are reported in Fig. 1. We can see that feedback is advantageous at any time, although its benefit increases with time, has a maximum at $t=0.4$, and then tends to decrease. Actually, the relative improvement $\left(\frac{C_{f b}-C_{0}}{C_{0}}\right.$ ), where $C_{f b}$ (respectively, $C_{0}$ ) is the concurrence with (respectively, without) feedback, plotted versus time (in the inset of Fig. 1) shows a maximum around $t=0.7$.

Another remarkable result is that the optimal feedback is achieved by the same values of $\alpha_{i}, \beta_{i}$ at any time. These values are reported in Table I and show a clear asymmetry between the action on the two subsystems.

\section{STABILIZING ENTANGLEMENT}

Suppose now we want to stabilize entanglement, i.e., we want to achieve the maximum entanglement at stationary state. In this case we also need an interaction Hamiltonian, e.g.,

$$
H_{\text {int }}=2 J\left(\sigma_{1}^{\dagger} \sigma_{1}-I\right)\left(\sigma_{2}^{\dagger} \sigma_{2}-I\right)
$$

Then we have to solve (14) with null left-hand side. Subsequently, maximize the concurrence (16) over $\alpha_{i}, \beta_{i}$, and $\gamma_{i}$, as well as over $\alpha$ and $J$.

In the basis $\{|11\rangle,|10\rangle,|01\rangle,|00\rangle\}$ the involved master equation reads

$$
\begin{aligned}
0= & -\iota\left(\begin{array}{cccc}
2 J & -\iota \alpha & -\iota \alpha & 0 \\
\iota \alpha & -2 J & 0 & -\iota \alpha \\
\iota \alpha & 0 & -2 J & -\iota \alpha \\
0 & \iota \alpha & \iota \alpha & 2 J
\end{array}\right) \rho \\
& +\iota\left(\begin{array}{cccc}
2 J & -\iota \alpha & -\iota \alpha & 0 \\
\iota \alpha & -2 J & 0 & -\iota \alpha \\
\iota \alpha & 0 & -2 J & -\iota \alpha \\
0 & \iota \alpha & \iota \alpha & 2 J
\end{array}\right) \\
& +\left(\begin{array}{cccc}
A_{1} & 0 & 0 & 0 \\
0 & A_{1} & 0 & 0 \\
B_{1} & 0 & 0 & 0 \\
0 & B_{1} & 0 & 0
\end{array}\right) \rho\left(\begin{array}{cccc}
A_{1}^{*} & 0 & B_{1}^{*} & 0 \\
0 & A_{1}^{*} & 0 & B_{1}^{*} \\
0 & 0 & & 0 \\
0 & 0 & 0 & 0
\end{array}\right) \\
& +\left(\begin{array}{cccc}
A_{2} & 0 & 0 & 0 \\
B_{2} & 0 & 0 & 0 \\
0 & 0 & A_{2} & 0 \\
0 & 0 & B_{2} & 0
\end{array}\right) \rho\left(\begin{array}{cccc}
A_{2}^{*} & B_{2}^{*} & & 0 \\
0 & 0 & 0 & 0 \\
0 & 0 & A_{2}^{*} & B_{2}^{*} \\
0 & 0 & 0 & 0
\end{array}\right) \\
& -\frac{1}{2}\left(\begin{array}{cccc}
2 & 0 & 0 & 0 \\
0 & 1 & 0 & 0 \\
0 & 0 & 1 & 0 \\
0 & 0 & 0 & 0
\end{array}\right) \rho-\frac{1}{2} \rho\left(\begin{array}{cccc}
2 & 0 & 0 & 0 \\
0 & 1 & 0 & 0 \\
0 & 0 & 1 & 0 \\
0 & 0 & 0 & 0
\end{array}\right) .
\end{aligned}
$$

Writing again $\rho$ as (19), Eq. (32) can be put in the same form of (20), where, however, now $\mathbf{M}$ is defined as

$$
\mathbf{M}:=\left(\begin{array}{ccccccccrrrrrrr}
-\left|B_{1}\right|^{2}-\left|B_{2}\right|^{2} & -2 \alpha & 0 & -2 \alpha & 0 & 0 & 0 & 0 & 0 & 0 & 0 & 0 & 0 & 0 & 0 \\
r_{2}+\alpha & \chi_{1} & 4 J & 0 & 0 & -\alpha & 0 & -\alpha & -\alpha & 0 & 0 & 0 & 0 & 0 & 0 \\
i_{2} & -4 J & \chi_{1} & 0 & 0 & 0 & -\alpha & 0 & 0 & \alpha & 0 & 0 & 0 & 0 & 0 \\
r_{1}+\alpha & 0 & 0 & \chi_{2} & 4 J & -\alpha & 0 & 0 & -\alpha & 0 & 0 & 0 & -\alpha & 0 & 0 \\
i_{1} & 0 & 0 & -4 J & \chi_{2} & 0 & -\alpha & 0 & 0 & -\alpha & 0 & 0 & 0 & 0 & 0 \\
0 & r_{1}+\alpha & -i_{1} & r_{2}+\alpha & -i_{2} & -1 & 0 & 0 & 0 & 0 & -\alpha & 0 & 0 & -\alpha & 0 \\
0 & i_{1} & r_{1}+\alpha & i_{2} & r_{2}+\alpha & 0 & -1 & 0 & 0 & 0 & 0 & -\alpha & 0 & 0 & -\alpha \\
\left|B_{2}\right|^{2} & 2 \alpha & 0 & 0 & 0 & 0 & 0 & -\left|B_{1}\right|^{2} & 0 & 0 & -2 \alpha & 0 & 0 & 0 & 0 \\
0 & r_{1}+\alpha & i_{1} & r_{2}+\alpha & i_{2} & 0 & 0 & 0 & -1 & 0 & -\alpha & 0 & 0 & -\alpha & 0 \\
0 & i_{1} & -r_{1}-\alpha & -i_{2} & r_{2}+\alpha & 0 & 0 & 0 & 0 & -1 & 0 & -\alpha & 0 & 0 & \alpha \\
\alpha & 0 & 0 & \left|B_{2}\right|^{2} & 0 & \alpha & 0 & r_{1}+2 \alpha & \alpha & 0 & -\frac{1}{2} & -4 J & \alpha & 0 & 0 \\
0 & 0 & 0 & 0 & \left|B_{2}\right|^{2} & 0 & \alpha & i_{1} & 0 & \alpha & 4 J & -\frac{1}{2} & 0 & 0 & 0 \\
\left|B_{1}\right|^{2} & 0 & 0 & 2 \alpha & 0 & 0 & 0 & 0 & 0 & 0 & 0 & 0 & -\left|B_{2}\right|^{2} & -2 \alpha & 0 \\
\alpha & \left|B_{1}\right|^{2} & 0 & 0 & 0 & \alpha & 0 & \alpha & \alpha & 0 & 0 & 0 & r_{2}+2 \alpha & -\frac{1}{2} & -4 J \\
0 & 0 & \left|B_{1}\right|^{2} & 0 & 0 & 0 & \alpha & 0 & 0 & -\alpha & 0 & 0 & i_{2} & 4 J & -\frac{1}{2}
\end{array}\right) .
$$




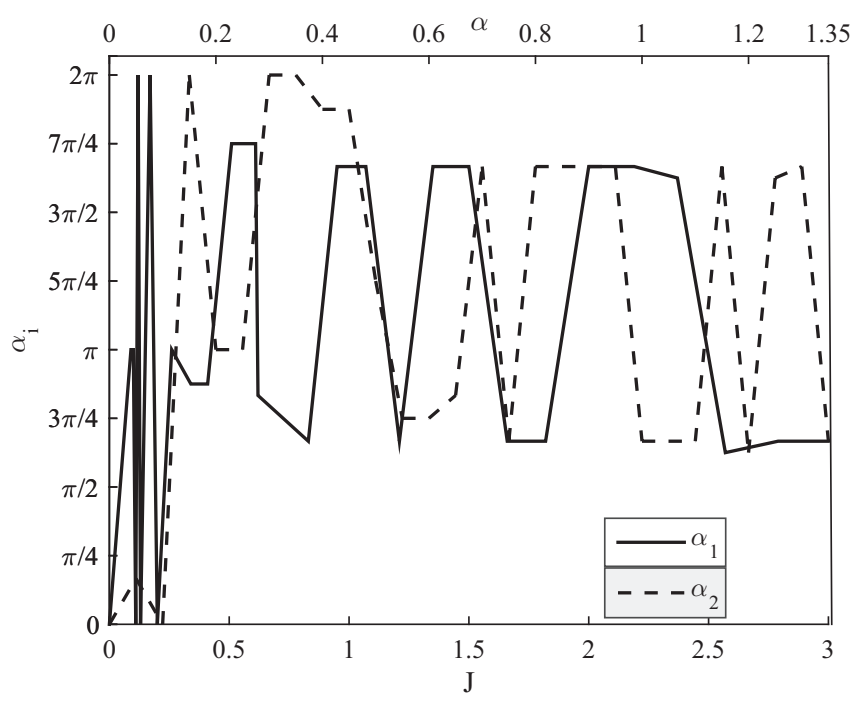

FIG. 5. Optimal values of $\alpha_{1}$ and $\alpha_{2}$ leading to maximum amount of entanglement along the curve $\alpha=\mathfrak{a}(J)$.

Also, in this case the dynamics results are independent from the $\gamma_{i}$ 's. Furthermore, we have no dependence from the initial state, hence the feedback action can again be considered universal.

The solution $\rho(\infty)$ is obtained analytically (not reported here for the sake of simplicity) and then optimization of concurrence has been pursued numerically by varying (for each value of $\alpha$ and $J$ ) $\alpha_{i}$ and $\beta_{i}$ in the range $0,2 \pi$ with step $\pi / 12$. Finally, the maximum value is taken as corresponding to optimal feedback and the value $\alpha_{i}=\beta_{i}=0$ is taken as corresponding to no feedback action. The concurrence $C(\rho(\infty))$ achieved with optimal feedback is plotted Fig. 2 vs $\alpha$ and $J$. The results have a mirror symmetry with respect to $\alpha=0$, so only positive values of $\alpha$ are considered.

Figure 3 demonstrates the difference between the concurrence $C(\rho(\infty))$ achieved with optimal feedback and that without feedback action.

Furthermore, in order to show the supremacy of our optimized feedback, in Fig. 4 we plotted the difference between the concurrence $C(\rho(\infty))$ achieved with optimal feedback and that with suboptimal feedback of Ref. [8].

By referring to Fig. 2 we may notice that for each value of $J$ there is an optimal value of $\alpha$ giving the largest concurrence. This determines a curve $\alpha=\mathfrak{a}(J)$ in the plane $J, \alpha$ along which we have maxima of concurrence. Then, in Figs. 5 and 6 we show the values of parameters $\alpha_{i}$ and $\beta_{i}$, respectively, that allow one to attain the maxima values of concurrence along a. As we can see, they oscillate and depend sensibly on the values of $\alpha$ and $J$.

\section{CONCLUSION}

We have addressed two main problems when controlling entanglement in two qubits dissipating into their own

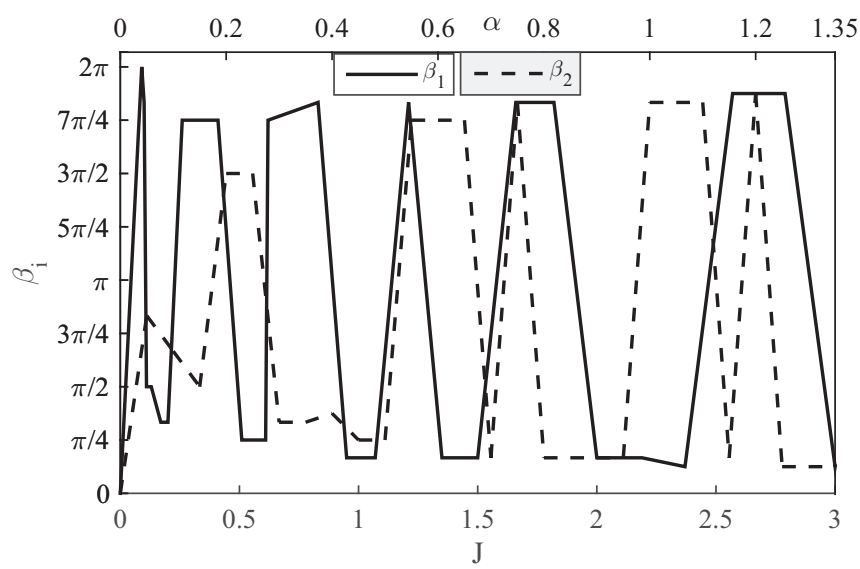

FIG. 6. Optimal values of $\beta_{1}$ and $\beta_{2}$ leading to maximum amount of entanglement along the curve $\alpha=\mathfrak{a}(J)$.

environments, namely, protecting initial entanglement and stabilizing entanglement. We have determined for both tasks optimal Markovian feedback control by resorting to analytical solutions of the dynamics as well as to numerical optimization of concurrence. The feedback actions are worked out in a way not depending on the initial two-qubit state, hence resulting as universal.

The present work fills the gap of Ref. [8] where a proof of principle of the effectiveness of Markovian feedback in stabilizing entanglement was given, but it was not optimal, as well as of Ref. [9] where the optimal Markovian feedback in protecting initial entangled states was derived in a way depending on the initial state and not continuous in time.

The proposed feedback can be realized in practice. In fact, we may notice that each feedback operator (11) representing a rotation in $\mathbb{R}^{3}$ can be considered as the composition of rotations along two directions ( $Z Y Z$ ). As such, it can be performed, for example, with a single laser pulse programed to be a chirped pulse with a temporal hole. The temporal hole in the middle of a chirped pulse induces a strong nonadiabatic evolution, which is a $Y$ rotation, amid an otherwise monotonic adiabatic evolution, a $Z$ rotation, due to the chirped pulse. The predicted behavior of the $Z Y Z$ decomposition can be experimentally verified with cold atomic qubits and as-programed femtosecond laser pulses [13]. Whether single qubits are in the excited or ground state can be detected, e.g., through ionization, using a frequency-doubled split-off of an unshaped laser pulse and a microchannel plate detector [14]. More generally, the found results could be helpful in designing experiments of entanglement control in settings such as cavity QED, trapped ions, and solid-state-based qubits [15].

The presented analysis can be extended rather easily to other interaction Hamiltonians, or even to more than two qubits. The exploitation of Bayesian (state-estimation-based) feedback control of two-qubit entanglement seems more challenging, following up the single-qubit control performed in Ref. [16].
[1] R. Horodecki, P. Horodecki, M. Horodecki, and K. Horodecki, Rev. Mod. Phys. 81, 865 (2009).
[2] P. Xu, X.-C. Yang, F. Mei, and Z.-Y. Xue, Sci. Rep. 6, 18695 (2016); A. Nourmandipour, M. K. Tavassoly, and M. Rafiee, 
Phys. Rev. A 93, 022327 (2016); T. J. Elliott, W. Kozlowski, S. F. Caballero-Benitez, and I. B. Mekhov, Phys. Rev. Lett. 114, 113604 (2015); S. M. Hashemi Rafsanjani and J. H. Eberly, Phys. Rev. A 91, 012313 (2015); F. Dolde, V. Bergholm, Y. Wang, I. Jakobi, S. Pezzagna, J. Meijer, Ph. Neumann, T. Schulte-Herbrueggen, J. Biamonte, and J. Wrachtrup, Nat. Commun. 5, 3371 (2014); S. Shankar, M. Hatridge, Z. Leghtas, K. M. Sliwa, A. Narla, U. Vool, S. M. Girvin, L. Frunzio, M. Mirrahimi, and M. H. Devoret, Nature (London) 504, 419 (2013); S. McEndoo, P. Haikka, G. de Chiara, M. Palma, and S. Maniscalco, Europhys. Lett. 101, 60005 (2013); F. W. Strauch, K. Jacobs, and R. W. Simmonds, Phys. Rev. Lett. 105, 050501 (2010); F. Platzer, F. Mintert, and A. Buchleitner, ibid. 105, 020501 (2010); X. Wang, A. Bayat, S. G. Schirmer, and S. Bose, Phys. Rev. A 81, 032312 (2010); X. Wang and S. G. Schirmer, ibid. 80, 042305 (2009); C. E. Creffield, Phys. Rev. Lett. 99, 110501 (2007).

[3] H. Rabitz, New J. Phys. 11, 105030 (2009).

[4] D. Dong and I. R. Petersen, IET Control Theory Appl. 4, 2651 (2010); B. Qi and L. Guo, Syst. Control Lett. 59, 333 (2010).

[5] H. M. Wiseman, Phys. Rev. A 49, 2133 (1994).

[6] J. Zhang, Y.-X. Liu, R.-B. Wu, K. Jacobs, F. Nori, Phys. Rep. 679, 1 (2017),
[7] S. Mancini and H. M. Wiseman, Phys. Rev. A 75, 012330 (2007); A. Serafini and S. Mancini, Phys. Rev. Lett. 104, 220501 (2010).

[8] S. Mancini and J. Wang, Eur. Phys. J. D 32, 257 (2005).

[9] M. Rafiee, A. Nourmandipour, and S. Mancini, Phys. Rev. A 94, 012310 (2016).

[10] W. K. Wootters, Phys. Rev. Lett. 80, 2245 (1998).

[11] O. C. O. Dahlsten, C. Lupo, S. Mancini, and A. Serafini, J. Phys. A: Math. Theor. 47, 363001 (2014).

[12] K. Zyczkowski and H.-J. Sommers, J. Phys. A 34, 7111 (2001).

[13] H. Lee, Y. Song, and J. Ahn, Phys. Rev. A 96, 012326 (2017).

[14] Y. Song, H. G. Lee, H. Jo, and J. Ahn, Phys. Rev. A 94, 023412 (2016).

[15] D. Kim, S. G. Carter, A. Greilich, A. S. Bracker, and D. Gammon, Nat. Phys. 7, 223 (2011); J. Majer, J. M. Chow, J. M. Gambetta, J. Koch, B. R. Johnson, J. A. Schreier, L. Frunzio, D. I. Schuster, A. A. Houck, and A. Wallraff, Nature (London) 449, 443 (2007); M. Hirose and P. Cappellaro, ibid. 532, 77 (2016); H. P. Specht, Ch. Nölleke, A. Reiserer, M. Uphoff, E. Figueroa, S. Ritter, and G. Rempe, ibid. 473, 190 (2011).

[16] H. M. Wiseman, S. Mancini, and J. Wang, Phys. Rev. A 66, 013807 (2002). 